\title{
Perfil sociodemográfico del intermediario político: diferencias entre hombres y mujeres
}

\section{Sociodemographic profile of the political broker: differences between men and women}

\author{
Manuel Guerrero-Martelo \\ $\triangle$ manuelfguerrero@gmail.com \\ Universidad Cooperativa de Colombia, Facultad de Ciencias Sociales y Humanas, \\ Departamento de Psicología, Montería, Colombia

\section{Gonzalo Galván} \\ $\triangle$ galvan.patrignani@gmail.com \\ Universidad Cooperativa de Colombia, Facultad de Ciencias Sociales y Humanas, \\ Departamento de Psicología, Montería, Colombia. \\ Universidad Nacional de la Patagonia Austral, Facultad de Ciencias Humanas, \\ Departamento de Enfermería, Puerto San Julián, Argentina \\ Alejandro Granados-García \\ $\triangle$ alejogranadosgarcia@gmail.com \\ Pontificia Universidad Javeriana, Facultad de piscología, \\ Bogotá, Colombia
}

Recibido: 06-02-2018

Aceptado: 28-05-2018

Publicado: 01-06-2018

\section{RESUMEN}

Objetivo. el presente trabajo tiene como objetivo presentar las diferencias entre hombres y mujeres en una muestra de intermediarios políticos que consiguen votos para un patrón a través de incentivos materiales o simbólicos. Se comparan algunas características sociodemográficas de la participación en este tipo de trabajo, así como la presencia de estabilidad económica del hogar, la diferencia en la participación femenina y masculina por el tipo de zona rural o urbana, la proveniencia de una familia autoritaria, la confianza hacia el jefe o patrón político, y la confianza hacia el Estado. Método. Diseño no experimental, transversal descriptivo. Se obtuvo una muestra de 49 intermediarios políticos o brókeres, trece mujeres $(26,5 \%)$ y 36 hombres $(73,5 \%)$, de varios municipios del departamento de Córdoba. La edad de la muestra osciló entre los veinte y los setenta años ( $M=45,16 D E=11,60)$. Resultados. Se encontraron diferencias significativas entre hombres y mujeres en la variable estrato socioeconómico $(p \leq 0,01)$, la confianza en el estado $(p \leq 0,05)$ y el nivel educativo. No se encontraron diferencias significativas en el resto de variables. Conclusiones. hay pocas diferencias en la participación de hombres y mujeres como intermediarios políticos. Se sugiere que, para decrecer la actividad del clientelismo, se puede trabajar un enfoque diferencial entre mujeres y hombres, al aumentar el nivel de educación y de conocimiento hacia el Estado en las mujeres, así como también al ofrecerles oportunidades de trabajo en los estratos más bajos.

Palabras clave: intermediarios políticos, diferencias sexuales, características sociodemográficas.

Guerrero-Martelo, M., Galván, G., Granados-García, A. (2018). Perfil sociodemográfico del intermediario político: diferencias entre hombres y mujeres. Búsqueda, 5(20):75-87. DOI: https://doi.org/10.21892/01239813.393 


\section{ABSTRACT}

Objective. the present work has as objective to present the differences between men and women in a sample of political brokers that obtain votes for a patron through material and/ or symbolic incentives. Some sociodemographic characteristics of participation in this type of work are compared, as well as the presence of economic stability of the household, the difference in female and male participation by the type of rural or urban area, the origin of an authoritarian family, the confidence towards the boss or political boss, and trust towards the State. Method. descriptive, no experimental cross design. A sample of 49 political intermediaries or brokers was obtained, 13 women $(26.5 \%)$ and 37 men (73.5\%), from several municipalities of the department of Córdoba. The age of the sample ranged between 20 and 70 years $(M=45.16 S D=11.60)$. Results. significant differences were found between men and women in the socioeconomic stratum variable $(p \leq 0.01)$, trust in the state $(p \leq 0.05)$ and the level of education. No significant differences were found in the rest of the variables. Conclusions. there are few differences in the participation of men and women as political intermediaries. It is suggested that in order to decrease the activity of clientelism, a differential approach between women and men can be worked on, increasing the level of education and knowledge towards the State in women, as well as offering them job opportunities in the lower strata.

Keywords: political broker, sexual differences, sociodemographic characteristics

\section{INTRODUCCIÓN}

En el proceso de elección política, los integrantes de una colectividad eligen mediante el voto a los representantes políticos de su preferencia. En algunas regiones, este comportamiento de voto puede estar mediado por un intermediario o bróker (Hicken, 2011), el cual intercambia con el candidato político beneficios de diversos tipos con el objetivo de ayudarlo a ganar el cargo de representación política e incluso a gobernar (Stokes, Dunning, y Nazareno, 2013). El establecimiento del acuerdo informal donde el candidato ofrece al intermediario y a través de este al votante recursos que van desde dinero hasta puestos de trabajo a cambio de su ayuda y votos es conocido como "clientelismo político" (Hicken, 2011), un intercambio de recursos entre el candidato o representante político (patrón) y el intermediario y los votantes (clientes) que dificulta el ejercicio de la ciudadanía y la democracia (Zúñiga Herazo y Valencia López, 2016) en que el intermediario juega un papel principal (Auyero y Güneş-Ayata, 1997; Hicken, 2011; Madero, 2010).

El intermediario establece un arreglo con el patrón para colocar el voto del cliente a su favor a cambio de un pago (Holland y Palmer Rubin, 2015), y se hace mucho antes y mucho después del periodo electoral (Auyero y Benzecry, 2016; Llamas y Felipe, 2017), integrando el nivel micro del barrio y la comunidad hasta el nivel macro nacional al conectar al patrón con el cliente (Wolf, 2001). Como actividad de reproducción material de la economía de la unidad doméstica, es un trabajo comunitario o de organización colectiva (Coraggio, 2003) de tipo informal. Incluso, algunas actividades que pueden estar relacionadas con el intermediario son consideradas delitos en algunos países; como el ofrecimiento de los votos de un grupo a favor de un patrón o la compra de votos (Rueda, 2017), tipificados como los delitos de tráfico de votos y corrupción de sufragante en Colombia (Congreso de Colombia, 2017). 
Si entendemos la participación política como todas aquellas actividades de los ciudadanos que afectan la política (Deth, 2016), la intermediación sería un tipo de participación política convencional que se encuentra al borde de la participación política no-convencional (Delfino y Zubieta, 2010), la cual requiere ciertas características individuales para ejercerla como la cercanía social, estar motivado, tener manejo de grupo y tener relaciones interpersonales (Alcántara, 2015) que como parte de una estructura clientelar, implica una forma de comportamiento institucionalizado (Ortiz, Cárdenas y López, 2014), en el que el intermediario político es un cliente respecto al aspirante a representante político o patrón, lo cual implica tener una gran flexibilidad (Ocampo, 2014), y cuyas motivaciones principales están entre el dinero y el arraigo partidista (Larreguy, Olea, y Querubin, 2017).

Con estas características, la intermediación política es una actividad que puede ser realizada tanto por mujeres como por hombres. Sin embargo teniendo en cuenta la subordinación histórica de la fuerza, el conocimiento y la experiencia de las mujeres por parte de los hombres (Littlefield \& McLane-Davison, 2015); la exclusión histórica de la mujer de los procesos políticos (Ross, 2017); la entrada tardía de la mujer y consecuentemente la diferencia en su participación de los procesos de representación política (Coffé y Bolzendahl, 2010); el sesgo de que no tienen capacidad para la política (Bauer, 2017), y el señalamiento del menor interés y ambición de la mujer en los procesos de representación política (Schneider, Holman, Diekman, y McAndrew, 2016), posiblemente las mujeres activas como intermediarias tengan diferencias respecto a los hombres.

Por otro lado, las mujeres han incrementado su participación política en muchas formas más allá del ejercicio del voto (Fernández, 2008), aun cuando requieren más recursos políticos que los hombres para participar en cargos de representación política (Beauregard, 2014). Alguna características femeninas que pueden dificultar su participación política, como por ejemplo la alta sensibilidad se anula en relación a los hombres cuando se trata de un comportamiento político (Blinder y Rolfe, 2018). Por esta razón, el estudio de la participación de las mujeres como intermediarias políticas es una forma de aproximarse a la creciente participación de la mujer en la política.

Sin embargo, es necesario tener en cuenta que la variable sexo condiciona el acceso a los tipos de trabajo, así como la forma de desempeño y la gratificación de los mismo. Es más difícil tener un trabajo para el sexo femenino (Littlefield y Mc Lane-Davidson, 2015), dada la atribución de responsabilidades con el hogar y la familia que en muchas sociedades recaen sobre las mujeres (Carosio, 2010). Un estudio señaló que el 50\% de los ingresos de la unidad doméstica en la actualidad provienen de la mujer (Garcés et al., 2017), lo cual la señala como responsable de la mitad de la estabilidad económica, a pesar de sus múltiples responsabilidades.

El desempeño del trabajo femenino es influido también por el tipo de familia del que la mujer provenga. Las mujeres provenientes de familias de patrones democráticos son más perfeccionistas que las que vienen de familias de patrones autoritarios (Girardi, Velasco y Lambe, 2006), pero por otra parte las que provienen de patrones autoritarios participan más en política que en el caso de proveniencia de patrones no autoritarios (Singh y Dunn, 2015). Por otra parte, los hombres en general tienden a recibir más educación que las mujeres (Sánchez y Gamboa, 2013) y estas no se benefician de 
su experticia como los hombres cuando manejan personas (Anisman-Razin, Rozen, Halperin, y Saguy, 2018)we examined the role of leaders' domain-specific expertise and gender as affecting individuals' evaluation of proposals related to intergroup conflict. Across three studies, conducted in two different conflict-related contexts (IsraeliPalestinian conflict and the refugee crisis in Europe, por lo cual tendrán más ventaja en el mercado laboral que ellas. La diferencia entre zona rural y urbana también juega un papel importante; las mujeres de la zona rural no tienen ingresos propios y se dedican al trabajo doméstico, lo cual las hace dependientes y carentes de autonomía (Ballera y Parada, 2009). La confianza es otro diferenciador del trabajo entre hombres y mujeres; el estudio de Chiang y San Martin (2015) señala que las mujeres se desempeñan mejor cuando tienen confianza y están satisfechas con su jefe. A un nivel macro, se encontró que los hombres de estrato alto tienen más confianza en el Estado, mientras que el nivel más bajo de confianza en lo tienen las mujeres de estrato bajo (Delgadillo, 2010).

Se han encontrado pocos estudios de la diferencia entre hombres y mujeres que se desempeñan como intermediarios o políticos. Por este motivo, el presente trabajo tiene como objetivo describir las diferencias entre hombres y mujeres dedicados a la intermediación como un tipo de participación política. Para alcanzar este objetivo, se pretende presentar una descripción de las diferencias de sexo en varias de las características sociodemográficas de un grupo de intermediarios políticos, y otras características adicionales como la presencia de estabilidad económica del hogar, el tipo de zona (rural o urbana), la proveniencia de una familia autoritaria y dos tipos de confianza, una hacia el jefe o patrón, y la otras hacia el Estado. Los resultados permitirán conocer algunos aspectos de la participación del sexo femenino como intermediaria política en una actividad que tradicionalmente ha sido ejercida por el sexo masculino, con algunas excepciones (Hernández, 2009) e indagar el perfil sociodemográfico de la mujer activa en la redes del clientelismo, en las que el intermediario juega un papel fundamental (Hicken, 2011). Este conocimiento permitirá formular programas y políticas públicas para controlar el clientelismo mediante la focalización en la diferencia entre hombres y mujeres, y la mejoría del ejercicio de la democracia.

\section{MÉTODO}

Diseño empleado. diseño no experimental, transversal, descriptivo.

Instrumento para la recolección de la información. un instrumento ad hoc fue diseñado y utilizado para obtener los siguientes datos.

a. Variables sociodemográficas: municipio de nacimiento, edad, sexo, partido o corriente política, estado civil, estrato socioeconómico, número de hijos, trabaja sí o no, nivel educativo, ¿a cuál religión pertenece?

b. Variables relacionadas con intermediación política: ¿a qué edad empezó como gestor?, ¿alguien de su familia también es gestor?

c. Variables de la estructura familiar con posible respuesta (nada, poco, medio, mucho y extremo): ¿su hogar es estable económicamente? ¿Sus padres eran autoritarios y estaban pendientes de hacer cumplir las órdenes de la casa? 
d. Variables relacionadas con la confianza social con posible respuesta (nada, poco, medio, mucho y extremo): ¿usted considera que el candidato que apoya es el mejor? ¿Qué tanta confianza tiene en el Estado, el Gobierno y sus instituciones?

Participantes. se obtuvo de manera no probabilística una muestra de 49 intermediarios políticos o brókeres; trece mujeres (26,5\%) y 37 hombres $(73,5 \%)$, de varios municipios del departamento de Córdoba, provincia de Colombia (Apartadó=1; Ayapel=3; Canalete $=1$; Cereté $=3$; Chinú $=1$; Ciénega de Oro=4; Corozal=3; Lorica =4; Los Córdobas=1; Balcón del Cesar=1; Moñitos=2; Montería=12; Puerto Escondido=3; Sahagún=5; San Andrés $=1$; San Pelayo=2). La edad de la muestra osciló entre los veinte y los setenta años $(M=45,16 \mathrm{DE}=11,60)$, y se dividió en dos grupos compuestos por trece intermediarios urbanos $(26,5 \%)$ y 36 intermediarios rurales $(73,5 \%)$, de acuerdo al tipo de zona definida en el Plan de Ordenamiento Departamental. Los criterios de inclusión fueron firmar consentimiento informado, ser o haber sido intermediario político (bróker) al menos tres años, tener conocimiento de los conceptos de tráfico y compra de votos, y ser mayor de dieciocho años.

Procedimientos desarrollados. la recolección de datos se llevó a cabo durante dos meses en los municipios mencionados. El instrumento ad hoc fue administrado por estudiantes auxiliares de investigación previamente entrenados en psicología política en torno al tema de las redes clientelares e intermediarios políticos. Debido a que la actividad de intermediario político es de carácter informal y es realizada principalmente en comunidades con necesidades insatisfechas, fue necesario que estudiantes relacionados con estas comunidades localizaran a los participantes mediante la técnica de referidos con la familia nuclear y conocidos, para encontrar los participantes que cumplieran con los criterios de inclusión. Posteriormente se estableció un contacto empático y se les pregunto su interés en participar en el estudio; cuando la respuesta fue afirmativa, el consentimiento informado fue leído, explicado y firmado por cada uno de los participantes. Después se les hicieron las preguntas del instrumento ad hoc que el entrevistador tenía en un formato de respuesta único, y de ahí se pasó a la tabulación de los datos.

Análisis de datos y conflictos de interés. después de la recolección de los datos, se inició la tabulación. A partir de allí se creó una base de datos con los 49 participantes. Las técnicas estadísticas utilizadas para el análisis de datos fueron de tipo descriptivas y no paramétricas, debido al tamaño de la muestra: media, desviación estándar, U de Mann Whitney y Chi2.

El primer paso fue describir la media y la desviación estándar de las variables cuantitativas (Edad, edad de inicio como intermediario, número de hijos y estrato). En las variables tipo Likert, las opciones de respuesta fueron reemplazadas por números (nada $=0$; poco $=1 ;$ medio $=2 ;$ mucho=3; extremo=4), que correspondían a: estabilidad económica del hogar, confianza en el líder político, autoritarismo en la familia y confianza en el estado. En estas variables de tipo continua, la comparación entre mujeres y hombres fue hecha con U de Mann-Whitney mientras que para las variables categóricas (presencia de intermediario en la familia, nivel educativo, estado civil, religión, tipo de zona, trabajo formal) fue hecha con chi2.

El pack estadístico SPSS 23.0 (SPSS, 2015) se utilizó para realizar los análisis descritos $y$ el valor $\mathrm{p} \leq 0,05$ y valores por debajo del mismo fueron considerado como valor $p$ estadísticamente significativo. 


\section{RESULTADOS}

La muestra total (Tabla 1) estuvo conformada por 49 participantes; trece mujeres y 36 varones, con edad media de 45,16 (DE =11,60). De acuerdo al género se constituyeron dos grupos: mujeres y hombres. [ $n=13(26,5 \%)$ vs. $(n=36(73,5 \%)]$, respectivamente.

Tabla 1. Descriptivos de media y desviación estandar de edad, años como intermediario, número de hijos, estrato, estabilidad económica, confianza en el líder político, autoritarismo en la familia y confianza en el Estado.

\begin{tabular}{|c|c|c|c|c|c|c|c|c|}
\hline & Edad & $\begin{array}{l}\text { Años como } \\
\text { gestor }\end{array}$ & \# Hijos & Estrato & Est. econ. & Conf. lid. & Fam. aut. & Conf. Est. \\
\hline & $M(D E)$ & $M(D E)$ & $M(D E)$ & $M(D E)$ & $M(D E)$ & $M(D E)$ & $M(D E)$ & $M(D E)$ \\
\hline $\begin{array}{c}\text { Total } \\
(n=49)\end{array}$ & $\begin{array}{c}45,16 \\
(11,60)\end{array}$ & $\begin{array}{l}23,72 \\
(6,99)\end{array}$ & $\begin{array}{c}2,63 \\
(1,76)\end{array}$ & $\begin{array}{c}2,02 \\
(1,01)\end{array}$ & $\begin{array}{c}1,96 \\
(0,49)\end{array}$ & $\begin{array}{c}2,78 \\
(0,71)\end{array}$ & $\begin{array}{c}2,84 \\
(0.68)\end{array}$ & $\begin{array}{c}2,22 \\
(0,65)\end{array}$ \\
\hline $\begin{array}{l}\text { Mujeres } \\
(n=13)\end{array}$ & $\begin{array}{c}41,38 \\
(12,72)\end{array}$ & $\begin{array}{l}23,23 \\
(5,34)\end{array}$ & $\begin{array}{c}2,46 \\
(1,56)\end{array}$ & $\begin{array}{c}1,38 \\
(0,65)\end{array}$ & $\begin{array}{c}1,85 \\
(0,19)\end{array}$ & $\begin{array}{c}2,62 \\
(0,18)\end{array}$ & $\begin{array}{c}2,92 \\
(0,13)\end{array}$ & $\begin{array}{c}1,85 \\
(0,24)\end{array}$ \\
\hline $\begin{array}{l}\text { Hombres } \\
(n=37)\end{array}$ & $\begin{array}{c}46,52 \\
(11,04)\end{array}$ & $\begin{array}{l}23,90 \\
(7,56)\end{array}$ & $\begin{array}{c}2,69 \\
(1,84)\end{array}$ & $\begin{array}{c}2,25 \\
(1,02)\end{array}$ & $\begin{array}{c}2 \\
(0,06)\end{array}$ & $\begin{array}{c}2,83 \\
(0,12)\end{array}$ & $\begin{array}{c}2,81 \\
(0,12)\end{array}$ & $\begin{array}{c}2,36 \\
(0,81)\end{array}$ \\
\hline
\end{tabular}

Est. Econ.: estabilidad económica; Conf. Lid.:confianza en el líder; Fam. Aut.: Familia autoritaria; Conf. Est: Confianza en el Estado; M(DE): media y desviación estándar

Con relación a las diferencias significativas en las variables de la Tabla 1 , se encontró que no había diferencias significativas entre hombres y mujeres en cuanto a la edad, el año de inicio como intermediario, el número de hijos, la estabilidad económica de la familia, la confianza en el líder político y el autoritarismo en la familia (véase Tabla 2). Sin embargo, se encontraron diferencias significativas en la variable estrato socioeconómico $(p \leq 0,01)$ y la confianza en el Estado $(p \leq 0,05)$.

Tabla 2. Diferencias entre mujeres y hombres intermediarios políticos en edad, años como gestor, estrato, número de hijos, estabilidad ecónomica del hogar, confianza en el patrón, familia autoritaria y confianza en el Estado

\begin{tabular}{|c|c|c|c|c|c|}
\hline & & Rango promedio & U de Mann-Whitney & $\mathbf{Z}$ & Sig. (bilateral) \\
\hline \multirow{2}{*}{ Edad } & Mujer $(n=13)$ & 19,85 & \multirow{2}{*}{167} & \multirow{2}{*}{$-1,519$} & \multirow{2}{*}{0,129} \\
\hline & Varón $(n=36)$ & 26,86 & & & \\
\hline \multirow{2}{*}{ Años como gestor } & Mujer $(n=13)$ & 24,96 & \multirow{2}{*}{233} & \multirow{2}{*}{,- 011} & \multirow{2}{*}{0,991} \\
\hline & Varón $(n=36)$ & 25,01 & & & \\
\hline \multirow{2}{*}{ Estrato } & Mujer $(n=13)$ & 16 & \multirow{2}{*}{177} & \multirow{2}{*}{-3} & \multirow{2}{*}{$0,005^{*}$} \\
\hline & Varón $(n=36)$ & 28,25 & & & \\
\hline \multirow{2}{*}{ Número de hijos } & Mujer $(n=13)$ & 24,42 & \multirow{2}{*}{226} & \multirow{2}{*}{,- 173} & \multirow{2}{*}{0,863} \\
\hline & Varón $(n=36)$ & 25,21 & & & \\
\hline \multirow{2}{*}{$\begin{array}{l}\text { Estabilidad ecónomica } \\
\text { del hogar }\end{array}$} & Mujer $(n=13)$ & 22,46 & \multirow{2}{*}{201} & \multirow{2}{*}{,- 994} & \multirow{2}{*}{0,320} \\
\hline & Varón $(n=36)$ & 25,92 & & & \\
\hline \multirow{2}{*}{ Confianza en el patrón } & Mujer $(n=13)$ & 22,19 & \multirow{2}{*}{197} & \multirow{2}{*}{,- 963} & \multirow{2}{*}{0,336} \\
\hline & Varón $(n=36)$ & 26,01 & & & \\
\hline \multirow{2}{*}{ Familia autoritaria } & Mujer $(n=13)$ & 25,85 & \multirow{2}{*}{223} & \multirow{2}{*}{,- 306} & \multirow{2}{*}{0,759} \\
\hline & Varón $(n=36)$ & 24,69 & & & \\
\hline \multirow{2}{*}{ Confianza en el Estado } & Mujer $(n=13)$ & 19,5 & \multirow{2}{*}{162} & \multirow{2}{*}{$-1,945$} & \multirow{2}{*}{$0,052 *$} \\
\hline & Varón $(n=36)$ & 26,99 & & & \\
\hline
\end{tabular}

$* \mathrm{p} \leq 0,05 ; * * \mathrm{p} \leq 0,01$ 
Por otra parte, también se encontró que no hay diferencias significativas entre hombres y mujeres en las variables: presencia de gestor político en la familia, estado civil, religión, tipo de zona y si tenían un trabajo o actividad productiva adicional en ese momento. Solo se encontró diferencia significativa en el nivel educativo (ver Tabla 3).

Tabla 3. Diferencias entre mujeres y hombres intermediarios políticos en presencia de gestor en la familia, nivel educativo, estado civil, religión, tipo de zona y trabajo

\begin{tabular}{|c|c|c|c|c|c|}
\hline & & Total & Mujeres & Hombres & $X^{2}$ \\
\hline & & Total $(n=49)$ & $(n=13)$ & $(n=36)$ & \\
\hline \multirow{3}{*}{$\begin{array}{l}\text { Hay algún gestor político en } \\
\text { tu familia? }\end{array}$} & Sí & $23(46,9)$ & $6(12,2)$ & $17(34,7)$ & \multirow{2}{*}{0,947} \\
\hline & No & $26(53,1)$ & $7(14,3)$ & $19(38,8)$ & \\
\hline & Primaria & $3(6,1)$ & 0 & $3(6,1)$ & \multirow{4}{*}{$0,030 *$} \\
\hline \multirow{3}{*}{ Nivel educativo } & Secundaria & $13(26,5)$ & $4(8,2)$ & $9(18,4)$ & \\
\hline & Técnico & $13(26,5)$ & $7(14,3)$ & $6(12,2)$ & \\
\hline & Universitario & $20(40,8)$ & $2(4,1)$ & $18(36,7)$ & \\
\hline \multirow{4}{*}{ Estado civil } & Casado & $23(46,9)$ & $3(6,1)$ & $20(40,8)$ & \multirow{4}{*}{0,085} \\
\hline & Divorciado & $3(6,1)$ & $2(4,1)$ & $1(2)$ & \\
\hline & Soltero & $14(28,6)$ & $6(12,2)$ & $8(16,3)$ & \\
\hline & Union libre & $9(18,4)$ & $2(4,1)$ & $7(14,3)$ & \\
\hline \multirow{4}{*}{ Religión } & Católico & $36(73,5)$ & $9(18,4)$ & $27(55,1)$ & \multirow{4}{*}{0,794} \\
\hline & Cristiano & $10(20,4)$ & $3(6,1)$ & $7(14,3)$ & \\
\hline & Evangélico & $2(4,1)$ & $1(2)$ & $1(2)$ & \\
\hline & Ninguna & $1(2)$ & 0 & $1(2)$ & \\
\hline \multirow{2}{*}{ Tipo de zona } & Urbano & $13(26,5)$ & $4(8,2)$ & $9(18,4)$ & \multirow{2}{*}{0,686} \\
\hline & Rural & $36(73,5)$ & $9(18,4)$ & $27(55,1)$ & \\
\hline \multirow{2}{*}{ Trabajo } & Sí & $35(71,4)$ & $9(18,4)$ & $26(53,1)$ & \multirow{2}{*}{0,838} \\
\hline & No & $14(28,6)$ & $4(8,2)$ & $10(20,4)$ & \\
\hline
\end{tabular}

$* p \leq 0,05 ; * *=\leq 0,01$

\section{DISCUSIÓN}

Tanto en mujeres como hombres, la actividad como intermediario político comienza hacia la adultez temprana $(M=23,67)$ y continua al menos hasta la adultez intermedia $(M=45,16)$; el hecho de que en ambos sexos no hubiera diferencias en las dos variables señala que posiblemente en ambos empiezan y se consolidan en la misma etapa del ciclo evolutivo. Asimismo, también puede apuntar hacia el hecho de la intermediación política como actividad de reproducción de la vida material, lo cual está en concordancia con el aumento en la participación de la mujer en el trabajo y su rol compartido por igual en la estabilidad económica de la unidad doméstica (Garcés et al., 2017), así como con el aumento de la participación política de la mujer en los últimos años.

La diferencia entre hombres y mujeres en estrato socioeconómico y nivel educativo puede ser correlativa, dado que a menor estrato socioeconómico tiende a ser menor el nivel educativo. Según los resultados, las mujeres dedicadas a la intermediación política no tienen tanta educación, lo cual concuerda con la tendencia general de las mujeres a recibir menor educación (Sánchez y Gamboa, 2013), y son de menor estrato social que los hombres. Esto posiblemente se relacione también con realizar 
una actividad que tradicionalmente hacen los hombres. El riesgo de llevar a cabo esta actividad y abordar el espacio masculino empieza en las partes de la estructura social en las que hay mayores necesidades insatisfechas, especialmente en países en vías de desarrollo. La disponibilidad para el trabajo debido a la necesidad y la igualación en las responsabilidades entre hombres y mujeres hacia el hogar proyecta el trabajo de la mujer como intermediaria política, que no requiere de educación sino de otras capacidades relacionadas con la actividad social (Alcántara, 2015).

No hubo diferencia tampoco en el número de hijos, cuyo promedio fue de aproximadamente tres. Esto señala unas expectativas de vida parecidas en ambos sexos, lo que puede estar relacionado con el concepto de tener pocos hijos y poder darles mejores posibilidades. Tampoco hubo diferencias en lo relativo a la estabilidad económica del hogar, que puede estar relacionado con el punto anterior. Tampoco la hubo entre hombres y mujeres respecto a la confianza y satisfacción con el patrón, muy probablemente porque en la mayoría de los casos los intermediarios políticos no necesitan tener confianza en el representante político que apoyan sino unos beneficios materiales o simbólicos de fácil intercambio. Sin embargo, la diferencia significativa con menor confianza hacia el Estado presentada en las mujeres, y con mayor frecuencia de estrato socioeconómico bajo, confirma lo señalado por Delgadillo (2010), quien indica que hay menor confianza hacia el Estado en mujeres de estrato bajo. De esta forma, la baja confianza en el Estado puede estar relacionada con el poco acatamiento de las normas del mismo, y consecuentemente con comportamientos al borde de la ley como los que implica la actividad de intermediario político.

Las mujeres tienden menos que los hombres a realizar comportamientos ilegales y antisociales (Castro, Carbonell y Anestis, 2012). Sin embargo, la actividad de intermediación política se encuentra en el borde entre la legalidad y la ilegalidad, y puede ser que al no estar claro este límite las mujeres tiendan a hacerlo por igual.

Otro resultado es que no hay diferencias de sexo en la intermediación política de acuerdo al tipo de zona rural o urbana. Aunque en las zonas rurales tiende a haber especialización de rol, tal que a la mujer se le asignan las responsabilidades del hogar por tradición, en lo relativo a la intermediación política ambos géneros trabajan por igual en ambos tipos de zona. Sin embargo, la diferencia significativa de estrato socioeconómico y educación, ambos de menor nivel en las mujeres intermediarias políticas que en los hombres, puede estar relacionado con la necesidad de trabajo que, para mantener la estabilidad económica del hogar, y aun con menos educación que el hombre, conduce a las mujeres a hacer este tipo de actividad que no requiere necesariamente tener un buen nivel de educación.

Acerca de la proveniencia de familias autoritarias tampoco hay diferencias, probablemente porque en la estructura social de la región hay prevalencia de patrón autoritario, confirmado con la media total de autoritarismo $(M=2,84, \mathrm{DE}=0,68)$ sobre un puntaje máximo de 3 que fortalece la inferencia, lo cual también se relaciona con del estudio de Singh y Dunn (2015) quien señala que los patrones autoritarios implican mayor participación en la política. Tanto mujeres como hombres participan por igual en este caso. 
Por otra parte, la presencia de un gestor político en la familia no influye sobre la posibilidad de ejercer como hombre o como mujer como intermediario político. Son otros factores los que determinan este fenómeno. Tampoco el estado civil, que en su mayoría corresponde a individuos casados y solteros. La intermediación política parece que no depende de la formación de una familia. La religión tampoco fue un factor que se relacione con la diferencia entre hombres y mujeres. Diferentes tipos de religión influyen en la participación política de forma diferente (Driskell, Embry y Lyon, 2008) pero, al parecer, la religión católica a la cual pertenecían los participantes en su mayoría (seguida de la religión cristiana) influye de forma similar tanto en hombres como mujeres dedicados a la intermediación política en este caso.

Ni el tipo de zona ni la presencia de trabajo representaron tampoco una diferencia entre hombres y mujeres. Esto indica que hay igual distribución de mujeres intermediarias políticas tanto en el campo como en la ciudad. El campo no representa una diferencia en la participación de la mujer como intermediaria política, así como tampoco el tener otro trabajo -en su gran mayoría tanto hombres como mujeres de la muestra lo tenían-. Esto podría relacionarse con una alta motivación de logro y emprendimiento, de deseos de trabajar por parte de los intermediarios políticos de ambos sexos.

\section{CONCLUSIÓN}

En conclusión, tanto en hombres como mujeres, el perfil del intermediario político tiende a tener estabilidad, formar una familia, ser emprendedor y respetuoso de la religión y empezar en la adultez joven. Esto significa que más que ser hombre o mujer, lo importante es que se empieza como intermediario político hacia esta etapa en la que hay más reconocimiento del mundo adulto y más flexibilidad para aprender nuevas habilidades.

Según lo encontrado por Ondercin y Jones-White (2011), a menores niveles de conocimiento político, menor posibilidad de participar en la política. Para el caso de la presente investigación, es posible que haya más conocimiento político, lo cual no implica que el nivel de educación general aumente al mismo tiempo. Por esta razón, a pesar de que en la muestra las mujeres tienen menor nivel de educación, participan como intermediarias políticas igual que los hombres en muchos aspectos. Sin embargo, el menor nivel educativo y menor estrato pueden señalar cierta prevención en la mujer a participar de una actividad tradicionalmente masculina. Esto puede implicar que mujeres de mayor prestigio social y más educación se dediquen a tareas más tradicionales, que además no se encuentran en los bordes difusos de posibles comportamientos ilegales.

Los hombres tienden a mantener su confianza en el Estado y su actividad como intermediarios a pesar de que esta actividad bordea en ocasiones los límites de la legalidad. En el caso de las mujeres tienen menos confianza hacia el Estado y posiblemente esto les permite aproximarse a ese límite, porque se puede suponer que no consideran al Estado como fuente de autoridad.

Conforme a la tendencia mundial, la mujer está aumentando su participación en la política; aquí señalamos específicamente el aumento en la forma de intermediación, en la que, al parecer, participa en casi igualdad de condiciones que los hombres. Adicionalmente, la actividad de intermediación política puede ser una puerta también 
para la participación de la mujer en la unidad doméstica por ser una actividad informal que requiere habilidades sociales tan frecuentes e incluso mayores en mujeres que en hombres. En este nuevo espacio, al parecer hay circunstancias que facilitan a las mujeres actuar como intermediarias políticas, que son el tener poca educación, un estrato socioeconómico bajo y una menor confianza hacia el Estado.

Se concluye en general que tanto hombres como mujeres participan como intermediarios políticos en las redes clientelares, con ligeras diferencias. Los programas y leyes para combatir el clientelismo deben apuntar a una mayor información sobre el funcionamiento del Estado y sus beneficios específicamente en mujeres, así como aumentar los niveles educativos de estas y las oportunidades laborales y de estudio en los estratos menos privilegiados.

\section{Agradecimientos}

Agradecemos a Luis David Estupiñán, y María Claudia Bettin, integrantes del semillero Ágora de la Universidad Cooperativa de Colombia, sede Montería, y a Jésika López Romero por su colaboración en la tabulación de los datos. Artículo derivado de la investigación "Cultura política en víctimas de desplazamiento forzado en el barrio Cantaclaro de Montería", Universidad Cooperativa de Colombia, Id. 1490.

\section{REFERENCIAS}

Alcantara, M. (2015). El oficio de político. Madrid: Tecnos.

Anisman-Razin, M.; Rozen, R.; Halperin, E. y Saguy, T. (2018). Support for Leader's Decisions in Conflict and Negotiation: Women Do Not Benefit from Relevant Expertise While Men Do. Political Psychology, 39(3), 633-648. Doi: https://doi. org/10.1111/pops.12434

Auyero, J., \& Benzecry, C. (2016). La lógica práctica del dominio clientelista. Revista mexicana de ciencias políticas y sociales(226), 221-246.

Auyero, J. y Güneş-Ayata, A. (1997). ¿Favores por votos?: estudios sobre clientelismo político contemporáneo. Losada: Buenos Aires.

Ballera, M. y Parada, S. (2009). El empleo de las mujeres rurales. Roma: FAO-CEPAL.

Bauer, N.M. (2017). The Effects of Counterstereotypic Gender Strategies on Candidate Evaluations. Political Psychology, 38(2), 279-295. Doi: https://doi.org/10.1111/ pops. 12351

Beauregard, K. (2014). Gender, political participation and electoral systems: A crossnational analysis. European Journal of Political Research, 53(3), 617-634. Doi: https://doi.org/10.1111/1475-6765.12047 
Blinder, S. y Rolfe, M. (2018). Rethinking Compassion: Toward a Political Account of the Partisan Gender Gap in the United States. Political Psychology, 39(4), 889-906. Doi: https://doi.org/10.1111/pops.12447

Carosio, A. (2010). El trabajo de las mujeres: desigualdad, invisibilidad y explotación. Revista venezolana de estudios de mujer, 15(35), 7-13.

Castro, Y.; Carbonell, J. y Anestis, J. (2012). The influence of gender role on the prediction of antisocial behavior and somatization. The International journal of social psychiatry, 58(4), 409-416.

Chiang, M. \& San Martin, N. (2015). Análisis de la satisfacción y el desempeño laboral en los funcionarios de la Municipalidad de Talcahuano. Ciencia \& trabajo, 17(54), 159-165. Doi: https://doi.org/10.4067/S0718-24492015000300001

Coffé, H. y Bolzendahl, C. (2010). Same Game, Different Rules? Gender Differences in Political Participation. Sex Roles, 62(5-6), 318-333. Doi: https://doi.org/10.1007/ s11199-009-9729-

Coraggio, J. (2004). Economía del trabajo. En A. Cattani, La otra economía (págs. 151164). Editorial Altamira.

Congreso de Colombia. (2017, Agosto 17). Mediante la cual se modifica la ley 599 de 2000 y se dictan otras disposiciones para proteger los mecanismos de participación democrática. Ley 1864 de 2017.

Dawes, T.; Loewen, J. y Fowler, H. (2011). Social Preferences and Political Participation. The Journal of Politics, 73(3), 845-856.

Delfino, G.I. y Zubieta, E.M. (2010). Participación política: concepto y modalidades. Anuario de investigaciones, 17, 211-220.

Delgadillo, G. (2010). Confianza ciudadana en el gobierno. Monterrey. [Tesis doctoral inédita]. Universidad Autónoma de Nuevo León.

Deth, J.W. van. (2016). Political Participation. En: The International Encyclopedia of Political Communication (pp. 1-12). American Cancer Society. Doi: https://doi. org/10.1002/9781118541555.wbiepc171

Driskell, R.; Embry, E. y Lyon, L. (2008). Faith and Politics: The Influence of Religious Beliefs on Political Participation. Social Science Quarterly, 89(2), 294-314. Doi: https://doi.org/10.1111/j.1540-6237.2008.00533.x

Fernandez, A. (2008). Las mujeres en la política latinoamericana. Nueva sociedad, 218.

Garcés, C.R.; Soto, M.; Andrea, J.; Garcés, C.R.; Soto, M. y Andrea, J. (2017). La contribución económica de la mujer en los hogares chilenos. Convergencia, 24(74), 209-230. 
Girardi, C.I. y Velasco y Lambe, J. (2006). Padres autoritarios y democráticos y características de personalidad de estudiantes de licenciatura y posgrado. Revista Intercontinental de Psicología y Educación, 8(1). Recuperado de: http://www. redalyc.org/resumen.oa?id $=80280103$

Hernández, B. (2009). Women's Movement, Latin America. En: The International Encyclopedia of Revolution and Protest (pp. 1-7). American Cancer Society. Doi: https://doi.org/10.1002/9781405198073.wbierp1593

Hicken, A. (2011). Clientelism. Annual Review of Political Science, 14(1), 289-310. Doi: https://doi.org/10.1146/annurev.polisci.031908.220508

Holland, A.C. y Palmer-Rubin, B. (2015). Beyond the Machine: Clientelist Brokers and Interest Organizations in Latin America. Comparative Political Studies. Doi: https:// doi.org/10.1177/0010414015574883

Kam, D. (2012). Risk Attitudes and Political Participation. American Journal of Political Science, 56(4), 817-836.

Larreguy, H.; Marshall, J. y Querubín, P. (2016). Parties, Brokers, and Voter Mobilization: How Turnout Buying Depends Upon the Party's Capacity to Monitor Brokers. American Political Science Review, 110(1), 160-179. Doi: https://doi.org/10.1017/ S0003055415000593

Larreguy, H.; Olea, C.E.M. y Querubin, P. (2017). Political Brokers: Partisans or Agents? Evidence from the Mexican Teachers' Union. American Journal of Political Science, 61(4), 877-891. Doi: https://doi.org/10.1111/ajps.12322

Littlefield, M. y Mc Lane-Davidson, D. (2015). Global gender inequality. En: Encyclopedia of social work. National Association of Social Workers y Oxford Unversity Press USA.

Llamas, R. y Felipe, A. (2017). Intermediarios del voto: estudio exploratorio sobre la importancia de estos actores en las redes clientelistas en la ciudad de Cartagena. Recuperado de: http://repository.javeriana.edu.co/handle/10554/21877

Madero, M. (2010). Casas políticas y redes clientelares en Cartagena. Tesis de maestría. Bogotá D.C.: Facultad de economía, universidad nacional de Colombia.

Ocampo, G. (2014). Poderes regionales, clientelismo y estado. Bogotá: Odecofi-Cinep.

Ondercin, H.L. y Jones-White, D. (2011). Gender Jeopardy: What is the Impact of Gender Differences in Political Knowledge on Political Participation?. Social Science Quarterly, 92(3), 675-694. Recuperado de: https://doi.org/10.1111/j.15406237.2011.00787.x

Ortiz, C.; Cárdenas, R. y López, Z. (2014). Elementos teóricos de los clientelismos. Memorias, 12(1), 73-89. 
Quintelier, E.; Stolle, D. y Harell, A. (2012). Politics in Peer Groups: Exploring the Causal Relationship between Network Diversity and Political Participation. Political Research Quarterly, 65(4), 868-881.

Ross, K. (2017). Women and Politics. En: Gender, Politics, News (pp. 11-30). WileyBlackwell. Doi: https://doi.org/10.1002/9781118561652.ch2

Rueda, M.R. (2017). Small Aggregates, Big Manipulation: Vote Buying Enforcement and Collective Monitoring. American Journal of Political Science, 61(1), 163-177. Doi: https://doi.org/10.1111/ajps. 12260

Sanchez, M. y Gamboa, R. (2013). La vinculación entre educación y género. Actualidades investigativas en educación, 1-16.

Schneider, M.C.; Holman, M.R.; Diekman, A.B. y McAndrew, T. (2016). Power, Conflict, and Community: How Gendered Views of Political Power Influence Women's Political Ambition. Political Psychology, 37(4), 515-531. Doi: https://doi.org/10.1111/ pops. 12268

Singh, S.P. y Dunn, K. (2015). Authoritarianism, socioethnic diversity and political participation across countries. European Journal of Political Research, 54(3), 563581. Doi: https://doi.org/10.1111/1475-6765.12096

Stokes, S.C.; Dunning, T. y Nazareno, M. (2013). Brokers, Voters, and Clientelism. Nueva York: Cambridge University Press.

Wolf, E. (2001). Kinship, Friendship, and Patron-Client Relations in Complex Societies. Doi: https://doi.org/10.1525/california/9780520223332.003.0013

Zúñiga Herazo, L. y Valencia López, H. (2016). Ciudadanía y democracia en Cartagena: entre la exclusión social y el clientelismo político. Reflexión Política, 18(36). Recuperado de http://www.redalyc.org/resumen.oa?id=11049415011 